\title{
Quadratic behavior of fiber Bragg grating temperature coefficients
}

\author{
Gordon M. H. Flockhart, Robert R. J. Maier, James S. Barton, William N. MacPherson, \\ Julian D. C. Jones, Karen E. Chisholm, Lin Zhang, Ian Bennion, \\ lan Read, and Peter D. Foote
}

\begin{abstract}
We describe the characterization of the temperature and strain responses of fiber Bragg grating sensors by use of an interferometric interrogation technique to provide an absolute measurement of the grating wavelength. The fiber Bragg grating temperature response was found to be nonlinear over the temperature range $-70{ }^{\circ} \mathrm{C}$ to $80^{\circ} \mathrm{C}$. The nonlinearity was observed to be a quadratic function of temperature, arising from the linear dependence on temperature of the thermo-optic coefficient of silica glass over this range, and is in good agreement with a theoretical model. (C) 2004 Optical Society of America

OCIS codes: $\quad 060.0060,060.2300,060.2310,060.2370,060.2400,230.1480$.
\end{abstract}

\section{Introduction}

Bragg gratings are increasingly being used as optical fiber sensors for a wide range of applications, ${ }^{1}$ notably for strain and temperature measurements. The transduction mechanism is the dependence of the reflected Bragg wavelength on the measurand, and a compelling advantage is the compatibility of Bragg gratings with wavelength-division multiplexing. One of the primary physical parameters of interest is temperature, and fiber Bragg grating (FBG) sensors have been demonstrated for a number of applications. ${ }^{1}$

Extensive research has investigated the strain and temperature sensitivity of FBGs for use as optical

When this research was performed, G. M. H. Flockhart, R. R. J. Maier, J. S. Barton (j.s.barton@hw.ac.uk), W. N. MacPherson, and J. D. C. Jones were with the School of Engineering and Physical Sciences, Heriot-Watt University, Edinburgh EH14 4AS, UK. G. M. H. Flockhart is now with the Naval Research Laboratory, 4555 Overlook Avenue, S.W., Washington, D.C. 20375. When this research was performed, K. E. Chisholm, L. Zhang, and I. Bennion were with the Photonics Research Group, Electronic Engineering and Computer Science Division, Aston University, Birmingham B4 7ET, UK. K. E. Chisholm is now with Indigo Photonics, Ltd., Faraday Wharf, Holt Street, Birmingham B7 4BB, UK. I. Read and P. D. Foote are with BAE Systems, Advanced Technology Centre, Sowerby Building, FPC 267, P.O. Box 5, Bristol BS12 7QW, UK.

Received 14 July 2003; revised manuscript received 24 November 2003; accepted 9 February 2004.

0003-6935/04/132744-08 $\$ 15.00 / 0$

(C) 2004 Optical Society of America fiber sensors; however, the measured temperature and strain coefficients reported in the literature vary considerably. It is well known that the strain and temperature coefficients vary with wavelength ${ }^{2}$; however, the wavelength-normalized coefficients also show a spread of values. The wavelengthnormalized strain coefficient is observed to range from $0.70 \times 10^{-6} \mu \varepsilon^{-1}$ (Ref. 3) to $0.77 \times 10^{-6} \mu \varepsilon^{-1}$ (Ref. 2) for FBGs at approximately $1550 \mathrm{~nm}$; these differences could be due to variations in the strainoptic coefficients among different optical fibers. Also, the wavelength-normalized temperature coefficients cover a range of values from approximately $3 \times$ $10^{-6} \mathrm{~K}^{-1}$ (Ref. 4) to $8 \times 10^{-6} \mathrm{~K}^{-1}$ (Ref. 2) for FBGs with a nominal Bragg wavelength of $\sim 1550 \mathrm{~nm}$. The type and concentration of the dopant used in the core of the optical fiber to increase its refractive index is known to affect the thermo-optic coefficient of the glass. $^{5}$

Previous research has reported the nonlinear wavelength response as a function of temperature for FBGs at cryogenic temperatures owing to the nonlinear thermo-optic and thermal expansion coefficients of silica glass. ${ }^{6-8}$ A nonlinear temperature response was also suggested in a study of the temperature dependence of type I and type IIa gratings in highly doped $\mathrm{Ge}$ fibers $\left(\mathrm{GeO}_{2}\right.$ concentration $\geq 20$ mol. \%) with and without hydrogen loading. ${ }^{9}$ However, the authors observed a linear temperature response over the temperature range $296-576 \mathrm{~K}$; the nonlinear temperature response was indicated only by a single measurement at $77 \mathrm{~K}$. The authors of Refs. $6-8$ also reported measurements of hydrogenated single-mode 
telecommunication fiber (SMF28 fiber) that suggested a nonlinear wavelength response; however, only three measurement points were presented over the temperature range $77-573 \mathrm{~K}$. Therefore the origins of the nonlinearity were not clearly established; they may be previously reported nonlinearities at cryogenic temperatures ${ }^{6-8}$; alternatively, Flockhart et al. ${ }^{10}$ have observed changes in the temperature sensitivity that are due to buffer-recoating effects. A nonlinear temperature response has also been observed over the very wide temperature range $\sim 20{ }^{\circ} \mathrm{C}$ to $1150{ }^{\circ} \mathrm{C}$ for type IIa FBGs in $\mathrm{N}_{2}$-doped silica fibers. ${ }^{11}$ The dependence of temperature on wavelength was observed to increase from 0.013 to 0.0175 $\mathrm{nmK}^{-1}$ for a Bragg grating with a center wavelength of $\sim 1550 \mathrm{~nm}$ at room temperature.

The reported nonlinear temperature dependence of the Bragg wavelength is most likely due to the temperature dependence of the thermo-optic coefficient, provided that any other nonlinearities in the reported experimental systems are accounted for. The temperature dependence of the thermo-optic coefficient was observed to be linear over the temperature range $20{ }^{\circ} \mathrm{C}$ to $220{ }^{\circ} \mathrm{C}$ at a wavelength of $633 \mathrm{~nm}$ in unbuffered single-mode fiber by measurement of the fringe shift in a Fabry-Perot (FP) interferometer and a separate measurement of the thermal expansion coefficient. ${ }^{12}$ However, the magnitude of the average thermo-optic coefficient is known to decrease at longer wavelengths ${ }^{13}$; it is therefore possible that the temperature dependence of the thermo-optic coefficient will also be different at longer wavelengths than for measurements at $633 \mathrm{~nm}$. Thus it is desirable to carry out a direct experimental measurement of any nonlinear effect for FBG sensors at $1550 \mathrm{~nm}$. In practical sensor systems the temperature response is often assumed to be linear near room temperature. A typical temperature range for some FBG sensing and communications applications is $-70{ }^{\circ} \mathrm{C}$ to $+80^{\circ} \mathrm{C}$. Therefore, to permit accurate temperature or strain measurements to be performed, the wavelength dependence of the grating on temperature or strain must be well understood. In applications in which temperature is not the primary measurand of interest, the wavelength's dependence on temperature must be accurately known to allow suitable temperature compensation or discrimination to be made. ${ }^{14}$

In this paper we investigate the magnitude of the nonlinear wavelength response to temperature in detail over the wavelength range 1524-1573 $\mathrm{nm}$ and over a temperature range of $-70{ }^{\circ} \mathrm{C}$ to $+80^{\circ} \mathrm{C}$. We describe two techniques for interrogation of the Bragg wavelength, and we compare our data with an existing model for thermo-optic coefficients of glasses as a means of explaining the nonlinear temperature response. ${ }^{15,16}$ One interrogation technique is based on scanning interferometry for high-accuracy wavelength measurement. This technique is based on Fourier-transform spectroscopy, in which the signal is processed by use of Hilbert transforms, ${ }^{17,18}$ facilitating accurate measurements of the temperature and strain coefficients of an array of Bragg gratings. The second technique used is based on a system consisting of a scanning FP filter and a superluminescent broadband source at $1.55 \mu \mathrm{m}$ with a $-3-\mathrm{dB}$ bandwidth of $40 \mathrm{~nm}$. The gratings were all of type $\mathrm{I},{ }^{19}$ but they had different strengths (refractive-index modulation depth) and wavelengths $(\sim 1550 \mathrm{~nm})$. Our measurements show that the temperature response is nonlinear over the temperature range $-70{ }^{\circ} \mathrm{C}$ to $+80^{\circ} \mathrm{C}$, with a maximum deviation from linearity of $\sim 35 \mathrm{pm}$, which is equivalent to an error of $\sim 3.5^{\circ} \mathrm{C}$.

\section{Interferometric Fiber Bragg Grating Interrogation}

To investigate temperature and strain sensitivity we fabricated an array of three wavelength-division multiplexed FBGs from 1524 to $1573 \mathrm{~nm}$. The grating array was written into hydrogenated (200 bars for 20 $\mathrm{MPa}$ ) single-mode Ge-doped telecommunications fiber by two-beam holographic exposure by use of a frequency-doubled Ar-ion laser. The array consisted of a high-reflectivity $(\sim 100 \%)$ grating at 1524 $\mathrm{nm}$, a low-reflectivity $(\sim 37 \%)$ grating at $1549 \mathrm{~nm}$, and a high-reflectivity $(\sim 100 \%)$ grating at $1573 \mathrm{~nm}$. The length of each grating was $\sim 6 \mathrm{~mm}$; there was an $\sim 1$-mm separation between gratings. Immediately after inscription the grating array was recoated with DeSolite 950-200 splicing compound and annealed at $150{ }^{\circ} \mathrm{C}$ for $24 \mathrm{~h}$ to ensure the spectral stability of the gratings at the desired maximum operating temperature of $80^{\circ} \mathrm{C}$.

The grating array was housed in a purpose-built, automated temperature and strain test rig. The test rig was designed to simulate the environmental conditions that the gratings would experience in typical applications such as structural monitoring over a temperature range of $-30{ }^{\circ} \mathrm{C}$ to $+80^{\circ} \mathrm{C}$ and a strain range of $0-2000 \mu \varepsilon$. The temperature-cycling test rig consisted of two cascaded Peltier heat pumps driven and controlled by a $40-\mathrm{W}$ proportional, integral, and derivative (PID) thermoelectric controller (Wavelength Electronics, Model LFI-3551). A large actively cooled heat sink was required for dissipating the heat transferred during cooling, thus allowing the low-temperature extreme to be achieved. The recoating material was removed to eliminate any possible thermal effects caused by the buffer material, as were previously observed. ${ }^{10}$ The FBG array was located in a 1-mm-wide channel between two 2-mmthick aluminum plates, which were instrumented with thermistors for the PID control, and three Class B platinum $100-\Omega$ resistance thermometers (RTDs) for accurate temperature measurement across the plate. To ensure good thermal conductivity between the aluminum plates and the FBGs, the array was embedded in silicone grease with a thermal conductivity of $0.16 \mathrm{~W} \mathrm{~m}^{-1} \mathrm{~K}^{-1}$; silicone grease was also chosen to minimize any strain transfer between the grease and the FBG array. The Peltier elements and the aluminum plates were thermally insulated from the outside environment by $\sim 40 \mathrm{~mm}$ of polystyrene. To minimize condensation and freezing of wa- 


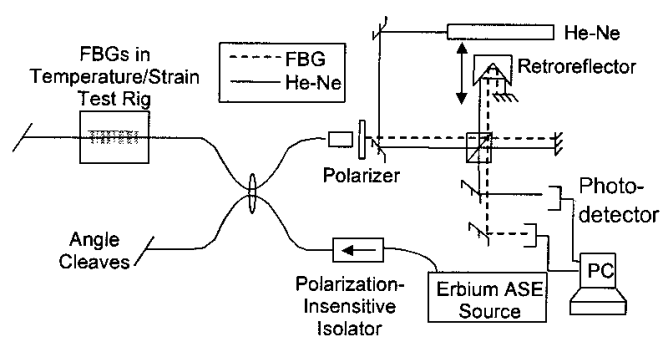

Fig. 1. Diagram of the experimental setup.

ter vapor at temperatures below the dew point, silica gel was used in the temperature-controlled chamber to provide a dry atmosphere.

The test rig was also designed to allow the fiber Bragg gratings to be strained. The optical fiber was clamped between two 0.75-mm-thick compliant sheets attached to brass plates. A clamping length of $15 \mathrm{~mm}$ was used, and, with sufficient clamping pressure, no slippage was observed during these experiments. One end of the fiber was clamped to a Melles-Griot Nanostepper translation stage, which was used to strain the FBG array longitudinally. The other end was clamped to a 2-kg load cell to monitor directly the load on the fiber. To determine the strain on the fiber from the applied load, one must know the Young modulus of the fiber; however, it is possible to calibrate directly the output of the load cell to the applied strain by measuring the load cell's output versus the translation stage's displacement for two different known lengths of fiber. Using this technique, we found the calibration constant to convert the load cell voltage output into applied strain to be $338.3 \pm 3 \mu \varepsilon \mathrm{V}^{-1}$.

Fourier-transform spectroscopy was used to measure the FBG's amplitude spectra from all three gratings simultaneously. The experimental setup is shown in Fig. 1. Light from a broadband Er amplified spontaneous emission (ASE) source $\left[P_{\text {out }}=15\right.$ $\mathrm{dBm}, \lambda_{(-3 \mathrm{~dB})}=1527-1562 \mathrm{~nm}$ ] illuminated the FBG array via a polarization-independent optical isolator and a wideband coupler (3- $\mathrm{dB}$ coupler with $\pm 0.3-\mathrm{dB}$ uniformity at $1550 \pm 40 \mathrm{~nm}$ ). The FBG array was fusion spliced to one arm of the coupler, and the distal ends of the two coupler arms were angle cleaved to prevent Fresnel reflections from propagating back along the fiber. The light reflected from the gratings was collimated and launched into a scanning Michelson interferometer. In one arm of the interferometer a hollow retroreflector, mounted upon a Melles-Griot Nanostepper translation stage, folded the beam onto the end mirror. $\mathrm{A} \mathrm{He}-\mathrm{Ne}$ laser beam, $\lambda=1523.1 \mathrm{~nm}$, propagated collinearly with the light from the gratings and was used to calibrate the optical path difference (OPD) scan to provide an absolute wavelength reference for the measurements. An OPD scan of $87.4 \mathrm{~mm}$, corresponding to $2^{18}$ samples, was used for the Fourier transform, thus giving a spectral resolution of $27.5 \mathrm{pm}$ at $1550 \mathrm{~nm}$; however the Bragg wavelengths were demodulated with bet- ter resolution when Hilbert transform signal processing was used. ${ }^{17}$

\section{Results and Discussion}

\section{A. Temperature Characterization}

A temperature range of $-30{ }^{\circ} \mathrm{C}$ to $80{ }^{\circ} \mathrm{C}$ was investigated. Narrow-range thermistors were used to improve the thermal stability of the PID control loop; therefore the temperature range was split into three subranges: $-30{ }^{\circ} \mathrm{C}$ to $5{ }^{\circ} \mathrm{C}, 7.5^{\circ} \mathrm{C}$ to $42.5^{\circ} \mathrm{C}$, and $45^{\circ} \mathrm{C}$ to $80{ }^{\circ} \mathrm{C}$. Three repeated temperature cycles were carried out in each subrange by use of a temperature interval of $2.5^{\circ} \mathrm{C}$ and a settling time of 15 min to allow the aluminum plates and the FBGs to achieve thermal equilibrium.

At each temperature set point an interferogram was acquired and the temperature was measured by both a Pt RTD and a thermistor. The standard deviation of the thermistor temperature measurements at each set point was approximately $0.05{ }^{\circ} \mathrm{C}$, indicating good thermal stability. The interferograms were recalibrated with the superimposed reference $\mathrm{He}-\mathrm{Ne}$ interferometer, and a Fourier transform was applied, resulting in the amplitude spectra of the FBGs. The FWHM of the three gratings' spectra were measured to be $0.8,0.2$, and $0.9 \mathrm{~nm}$ for the 1524-, 1549-, and 1573-nm gratings, respectively. Bragg wavelength can be calculated by a number of techniques such as peak detection and curve fitting; however, the spectra of the strong gratings are not ideally suited to either of these techniques, as they exhibit a flat-topped response. Therefore the center wavelength of each grating was calculated by the Hilbert transform technique. ${ }^{10}$ The measured temperature response for the 1549-nm FBG is shown in Fig. 2. For each grating a linear least-squares (LSQ) fit was made to the measured temperature response and the residual to the fit was calculated; the residual for the $1549-\mathrm{nm}$ FBG is shown in Fig. 2(b). The residual clearly shows higher-order structure, and similarly shaped residuals were also observed for the 1524- and 1573-nm FBGs in the array.

Second-order polynomial LSQ fits were made of the measured temperature responses, and the residuals to the fits were calculated. The residual to the second-order fit for the 1549-nm FBG is shown in Fig. 2(b). The residuals showed no higher-order structure present and showed a general scatter about zero, thus indicating a good fit. Regression analysis was used to determine the standard errors of the fit and to calculate $95 \%$ confidence belts for the fit; these values are also shown in Fig. 2(b) to indicate the quality of the fit and the magnitude of the observed scatter. The coefficients and the standard errors of the coefficients were calculated from the least-squares fits and are listed in Table 1 . The $R^{2}$ regression coefficient for each FBG indicates an excellent fit $\left(R^{2}=1\right.$ indicates a perfect fit); this was verified by the small magnitude of the standard error for each coefficient. The fitted coefficients for all the gratings showed good correlation and showed a slight increase in relation to 

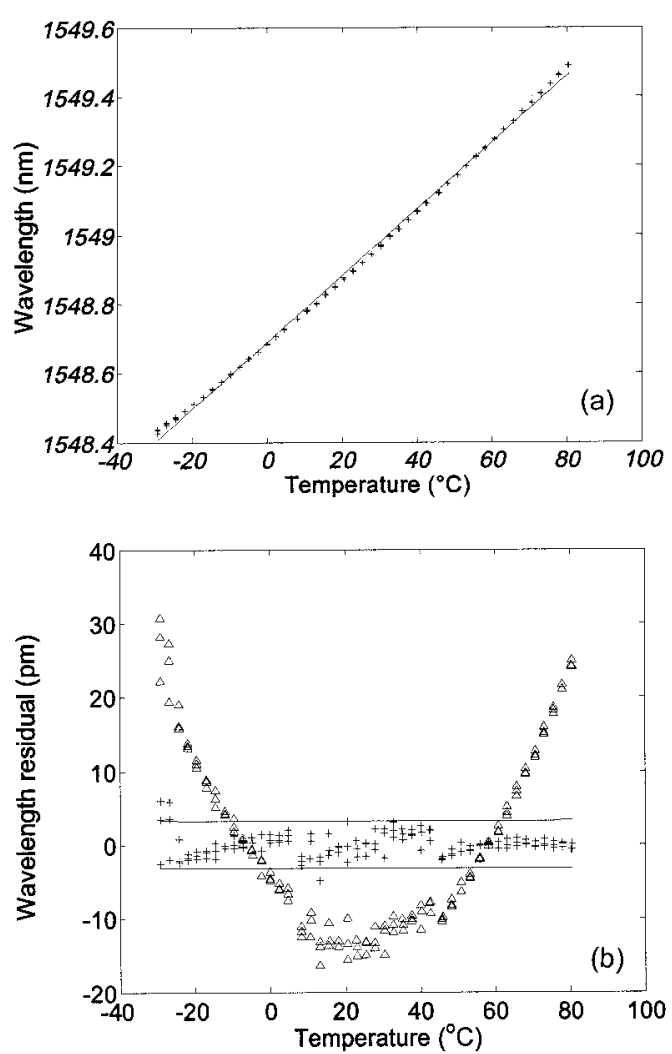

Fig. 2. (a) Temperature response of the 1549-nm FBG: measured wavelength shift (crosses) and LSQ fit (solid curve). (b) Residual to the linear fit (triangles) and second-order fit (crosses) with $95 \%$ confidence belts.

the Bragg wavelength. This indicates the dispersive nature of the thermo-optic coefficient; however care must be taken, as the magnitude of this increase for the second-order term for the 1549- and 1573-nm FBGs can be accounted for by the error of the fitted coefficient.

Possible experimental causes of the nonlinearity have been identified as FBG convolution with the source spectrum, nonlinear temperature measurement, presence of a temperature gradient across the aluminum plate in the test rig, and an artifact of signal processing. We believe that these causes can all be eliminated. First, as all three FBGs lie in different regions of the source spectrum, the convolution will be different for each one. Although the measured spectra of the 1524- and 1573-nm FBGs are affected by the slope of the source spectrum, the change in the convolution as the FBG spectrum moved with temperature was investigated and found to have a negligible effect on the measured temperature response. The source spectrum was nearly flat for the 1549-nm FBG; thus it had a minimal affect on the measured wavelength. Second, the measured temperature response was similar for Pt RTDs and thermistors; the thermistors used for this research had a large negative thermal coefficient, the opposite of the Pt RTD response. The effect due to the presence of a temperature gradient across the plate can also be eliminated. Each grating has a different physical location within the plates and thus experiences a slightly different temperature. At the lowand high-temperature extremes the two gratings nearer the edges of the plates will experience a temperature closer to the ambient temperature. If this difference is significant a residual of a different form from that observed would be produced; also, the 1549-nm FBG, which is located centrally within the aluminum plates, would be least affected by any temperature gradient. Finally, signal processing can be eliminated as a cause of nonlinearity, as the calculated wavelengths for the strain cycling experiments, which are detailed below, do not show the same residual. The quadratic residual is therefore believed to be due to a nonlinear temperature response of the grating.

\section{B. Secondary Experiment}

To confirm the observed nonlinearity we performed an independent experiment on a single FBG, using a different wavelength interrogation technique and a different temperature cycling setup. The FBG was fabricated in SMF28 fiber with a center Bragg wavelength of $1557 \mathrm{~nm}$, a FWHM of $0.3 \mathrm{~nm}$, and a reflectivity of $\sim 50 \%$. The FBG was immersed in an isopropanol (IPA) and dry-ice slush bath, which allowed a low-temperature extreme of $-70{ }^{\circ} \mathrm{C}$ to be reached. To ensure absolute strain-free mounting, we held the fiber at a single location just short of the FBG, with the remaining short length of fiber floating in the bath. A schematic of the experimental setup is shown in Fig. 3. A two-stage bath was used, with the outer bath stirred strongly to ensure homogeneous temperature distribution and the inner bath stirred gently to minimize convection currents. A small heater was used to raise the temperature of the outer slush bath. The FBG and a Pt RTD located in close proximity to the FBG were wrapped into a thin Ai foil to minimize local temperature gradients. The FBG was illuminated by a superluminescent diode source [SLD; $\mathrm{P}_{\text {out }}=8.5$ $\left.\mathrm{dBm}, \lambda_{(-3 \mathrm{~dB})}=1520-1570 \mathrm{~nm}\right]$ via an optical fiber

Table 1. Calculated Coefficients and Standard Errors for LSQ Second-Order Fits to the FBGs' Temperature Responses and the $R^{2}$ Regression Coefficients

\begin{tabular}{ccccc}
\hline$\sim \lambda_{\text {FBG }}(\mathrm{nm})$ & $a \times 10^{-6}\left(\mathrm{~nm} \mathrm{~K}^{-2}\right)$ & $b \times 10^{-3}\left(\mathrm{~nm} \mathrm{~K}^{-1}\right)$ & $c(\mathrm{~nm})$ & $R^{2}$ \\
\hline 1524 & $12.22 \pm 0.14$ & $8.8049 \pm 0.0082$ & $1523.98598 \pm 0.00017$ & 0.999976 \\
1549 & $12.73 \pm 0.15$ & $9.0103 \pm 0.0086$ & $1548.68187 \pm 0.00018$ & 0.999974 \\
1573 & $12.83 \pm 0.17$ & $9.1044 \pm 0.0099$ & $1572.79187 \pm 0.00021$ & 0.999965 \\
\hline
\end{tabular}




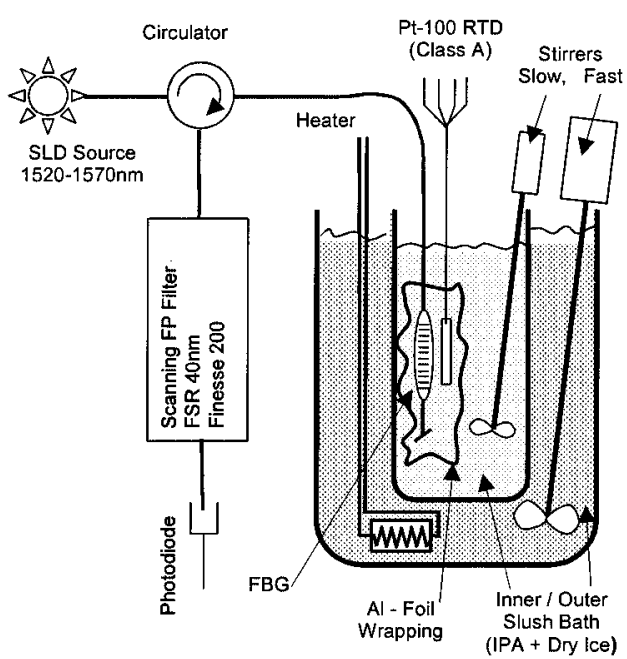

Fig. 3. Experimental setup for the secondary temperature characterization experiment.

circulator; light reflected from the grating was passed back through the circulator to a FP filter (Queensgate DMC 300). The FP filter [free spectral range (FSR), $40 \mathrm{~nm}$; finesse, 200] was temperature stabilized to $\pm 0.1{ }^{\circ} \mathrm{C}$.

With this experimental configuration the temperature is not actively controlled but changes slowly; the heater is used to raise the temperature of the slush bath above ambient. The duration of the wavelength scan of the FP filter across the grating spectrum is typically $3 \mathrm{~s}$, and the temperature of the Bragg grating during the scan is measured with the Pt RTD. A second-order polynomial, fitted to the convolved spectrum above the $-3-\mathrm{dB}$ level, is used to calculate the centroid location of the Bragg reflection peak. The measured temperature response of the FBG from $-70{ }^{\circ} \mathrm{C}$ to $75^{\circ} \mathrm{C}$ is shown in Fig. 4. As in the previous experiment described above, a linear LSQ fit was made to the measured temperature response and the residuals to first- and second-order fits were calculated and are shown in Fig. 4(b). As in the principal experiment, the residual to the firstorder fit clearly shows higher-order structure of a quadratic nature. A second-order fit confirms this; however, further structure is still evident, but this is due to a temperature variation between the FBG and the Pt RTD when the temperature of the heater was changed. The cause of the temperature variation is the different thermal time constants of the two sensors as a result of their different sizes; the thermal time constant of the FBG is much less than that of the Pt RTD.

The observation of the quadratic temperature response with this secondary experiment confirms our initial observation, and the nonlinearity was not an artifact of our experimental system. For most sensing applications the temperature response of FBGs is assumed linear over this temperature range; however, this nonlinearity cannot be neglected for sensing systems that require measurement accuracies
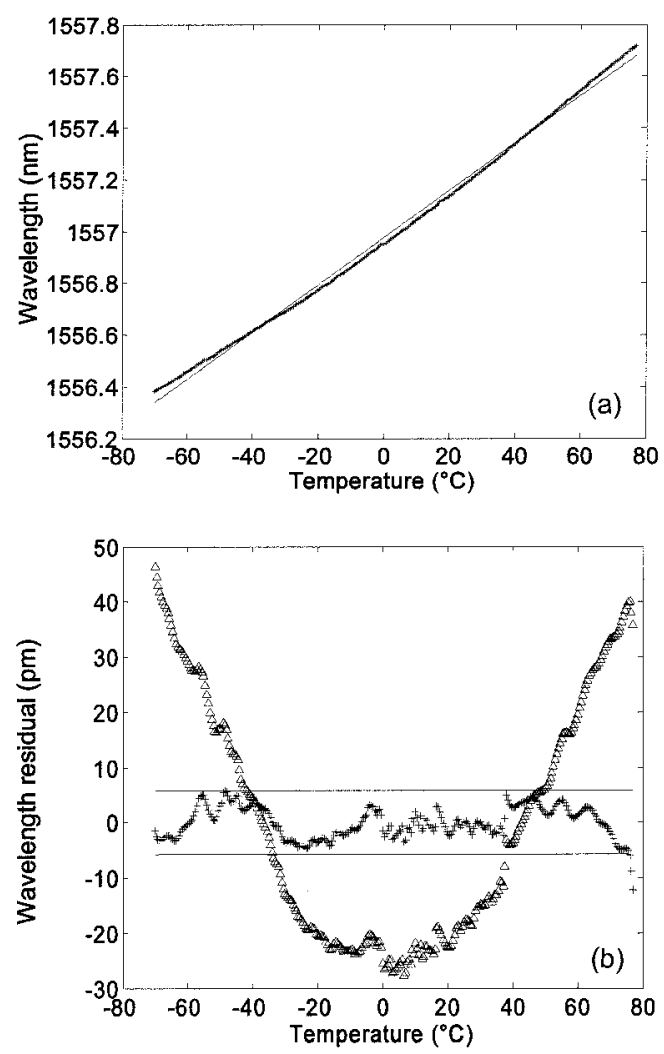

Fig. 4. (a) Temperature response of the 1557-nm FBG: measured data (darker curve) and linear LSQ fit (lighter curve). (b) Residual to the linear fit (triangles) and second-order fit (crosses) with $95 \%$ confidence belts.

better than $\pm 35 \mathrm{pm}$, as indicated by the deviation from linearity shown in Fig. 4.

\section{Model of the Thermo-Optic Coefficient}

We have applied a model developed by Ghosh to explain the behavior of the thermo-optic coefficient $(\mathrm{d} n$ / $\mathrm{d} T$ ) of optical glasses; this model was applied to silicate glasses ${ }^{15}$ and to standard optical glasses. ${ }^{16}$ The model of standard optical glasses is based on measurements of the thermo-optic coefficient and the thermal expansion of four National Institute of Standards and Technology standard reference material glasses. ${ }^{20}$ One of these materials is silica, which is the main host material used in the fabrication of optical fibers. The model developed by Ghosh is based on the temperature dependence of the excitonic bandgap, an isentropic bandgap, and the thermal expansion coefficient of the glass. Ghosh considers the thermo-optic coefficient to be dependent on both electronic transitions and optical phonons. The thermooptic coefficient is described by ${ }^{16}$

$$
2 n \frac{\mathrm{d} n}{\mathrm{~d} T}=\left(n_{0}^{2}-1\right)\left(-3 \alpha R-\frac{1}{E_{\mathrm{eg}}} \frac{\mathrm{d} E_{\mathrm{eg}}}{\mathrm{d} T} R^{2}\right),
$$

where $n_{0}$ is the low-frequency refractive index in the infrared region, $\alpha$ is the coefficient of thermal expan- 
sion, $E_{\text {eg }}$ is the excitonic bandgap, and $R$ is the normalized dispersive wavelength, defined by

$$
R=\frac{\lambda^{2}}{\left(\lambda^{2}-\lambda_{\mathrm{ig}}^{2}\right)},
$$

where $\lambda_{\mathrm{ig}}{ }^{2}$ is the wavelength that corresponds to the isentropic bandgap, $E_{\mathrm{ig}}$. This bandgap has been observed to be unaffected by changes in temperature, ${ }^{21}$ whereas the excitonic bandgap decreases with temperature. Equation (1) shows that the thermo-optic coefficient depends on two terms; the first describes the contribution from the thermal expansion coefficient and the second describes the contribution from the temperature dependence of the excitonic bandgap. The first term is negative for silica, as the thermal expansion coefficient is positive within the temperature range that we are interested in. The contribution of this term is small, as the magnitude of $\alpha$ is of the order of $10^{-6} \mathrm{~K}^{-1}$. However, the temperature variation of $E_{\mathrm{eg}}$ is of the order of $10^{-4} \mathrm{eV} \mathrm{K}^{-1}$ and is normally negative for glasses. ${ }^{21}$ The contribution from the second term is therefore positive and is the dominant term, resulting in a positive thermooptic coefficient. By analysis of experimental refractive-index data, Ghosh calculated the temperature dependence of $E_{\text {eg }}$ and observed a nonlinear response, with $\mathrm{d} E_{\text {eg }} / \mathrm{d} T$ decreasing linearly with temperature; he calculated $\mathrm{d} E_{\text {eg }} / \mathrm{d} T$ over the temperature range $+25^{\circ} \mathrm{C}$ to $+115^{\circ} \mathrm{C}$.

Using the model and data presented by Ghosh, 16 we calculated the change in the thermo-optic coefficient with temperature over a temperature range of $-30{ }^{\circ} \mathrm{C}$ to $+80^{\circ} \mathrm{C}$ for the three grating wavelengths. We assumed that the linearity of $\mathrm{d} E_{\text {eg }} / \mathrm{d} T$ extends from $25^{\circ} \mathrm{C}$ to $-30{ }^{\circ} \mathrm{C}$, and the thermal expansion was assumed to be constant. The refractive index at each grating wavelength was calculated by use of temperature-dependent Sellmeier coefficients for fused silica. ${ }^{22}$ We then calculated the change in the grating's wavelength over the experimental temperature range, using the temperature-dependent thermo-optic coefficient, and the effective refractive index of the grating was assumed constant over the small wavelength change of the grating.

Figure 5(a) shows the theoretical change in wavelength calculated from the model and the experimental results for the 1549-nm FBG. To allow a graphic comparison of the residual to be made, as in the experimental measurements, we made a linear LSQ fit to the modeled data and calculated the residual, which is shown in Fig. 5(b). Excellent correlation can be observed between the change in wavelength with temperature and the shape and magnitude of the residual to a linear LSQ polynomial fit. A small variation in sensitivity can be observed between the measured and the modeled data; however, the nonlinearity of the temperature response agrees well with the experimental data. The variation in the sensitivity could be due to a difference between the thermal expansion coefficient used for the model and that of the fiber. The modeled data are also based on
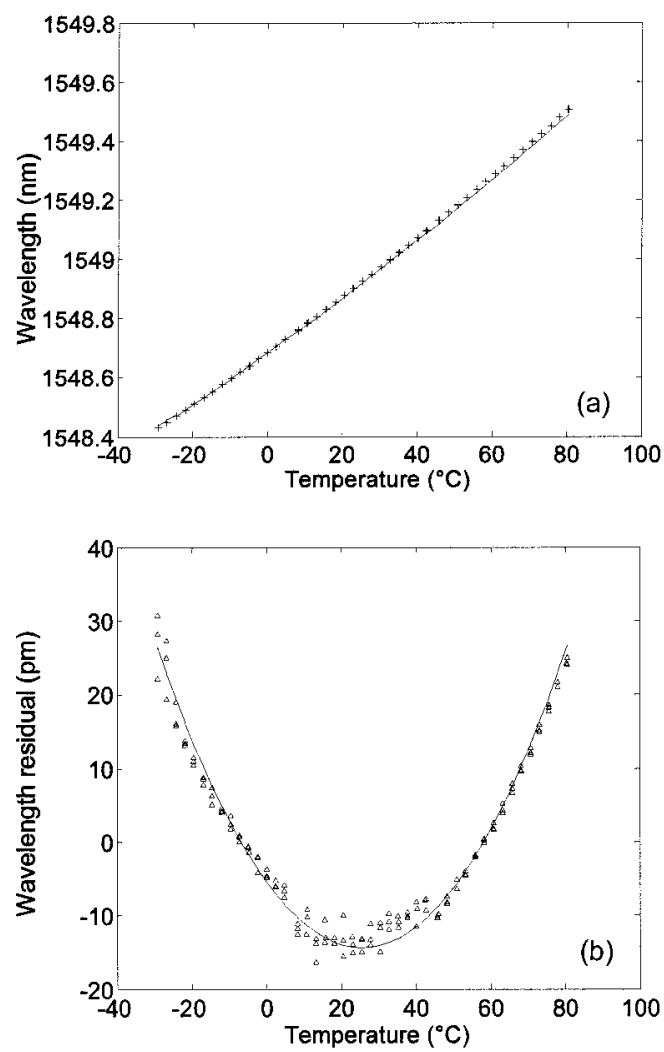

Fig. 5. (a) Temperature response of the 1549-nm FBG: measured wavelength change (crosses) and modeled data (solid curve). (b) Residual to the linear fit of experimental (triangles) and modeled (solid curve) data.

experimental measurements of the refractive index and the thermo-optic coefficient of fused silica; however, the core of the optical fiber is not pure fused silica but is doped to increase the refractive index. The exact core composition was not known. The Ge dopant will affect the thermo-optic coefficient; however, the dopant concentration is typically small $(<5 \%)$; therefore the temperature response of the optical fiber is assumed to be comparable to that of fused silica.

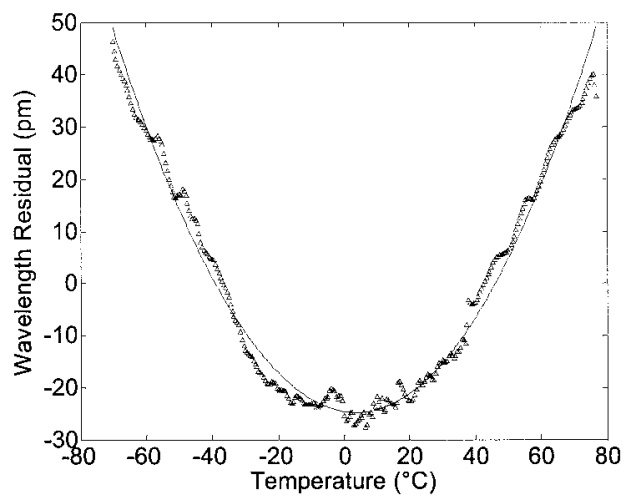

Fig. 6. Wavelength residual to the linear fit of the 1557-nm FBG temperature response (triangles) and residual of the calculated wavelength response for the model (solid curve). 

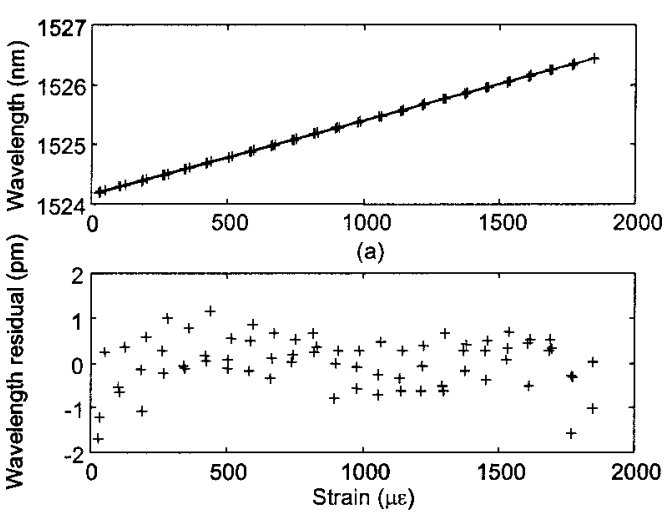

(b)
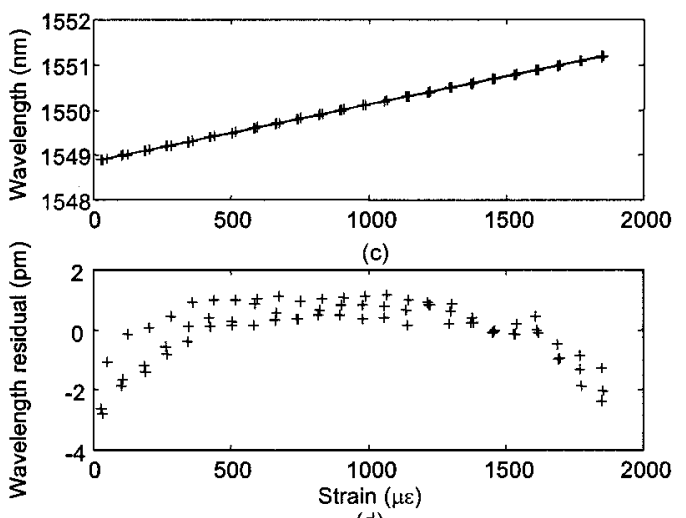

(d)

Fig. 7. (a) Strain response of the $1524-\mathrm{nm}$ FBG and (b) residual to the linear fit. (c) Response of the 1549-nm FBG and (d) residual to the linear fit.

We also applied the model to the measured wavelength response, using the secondary experiment over the extended temperature range of $-70{ }^{\circ} \mathrm{C}$ to $75^{\circ} \mathrm{C}$. As before, linear LSQ fits were made to the experimental data and the modeled data. The residuals to these fits show excellent correlation and are shown in Fig. 6.

\section{Strain Characterization}

The strain response of the FBGs in the array was also investigated. A Melles-Griot Nanostepper translation stage was used to strain the fiber, and the load on the fiber was measured directly with a $2-\mathrm{kg}$ load cell. The measured load was converted to strain by use of a calibration constant of the load cell that was previously measured. The temperature in the test rig was held constant at $20^{\circ} \mathrm{C}$, and the strain response was investigated over the range $0-2000 \mu \varepsilon$. The temperature stability was measured to be $\pm 0.01{ }^{\circ} \mathrm{C}$, which corresponds to an equivalent strain error of $\sim 0.1 \mu \varepsilon$. The strain responses of the 1524- and 1549-nm FBGs are shown in Fig. 7. As in the analysis of the temperature experiments, a linear LSQ fit was made to the strain response and the residual to the fit was calculated. The magnitude of the residuals is greatly reduced, although some structure is present; however, in general the residuals show an excellent repeatability, to within less than $5 \mathrm{pm}$. The residual for the 1524-nm FBG shown in Fig. 7(a) exhibits little structure, and the rms deviation of the LSQ fit is $0.57 \mathrm{pm}$, which suggests that the measurement technique is capable of subpicometer resolution. Regression analysis based on the linear LSQ fits was performed, and the calculated coefficients are listed in Table 2. The $R^{2}$ regression coefficient is approximately 1 for each strain response, indicating an excellent fit; this is also reflected in the small magnitude of the calculated standard error for each coefficient. The wavelength-normalized strain coefficients were calculated to be $0.81 \times 10^{-6} \mu \varepsilon^{-1}$ for all three FBGs in the array.

\section{Conclusions}

We have used Fourier-transform spectroscopy to provide absolute wavelength measurements to characterize the wavelength changes in fiber Bragg gratings with applied temperature or strain. We performed two independent experiments to investigate the linearity of the temperature response over a temperature range of $-70^{\circ} \mathrm{C}$ to $80^{\circ} \mathrm{C}$, which typically may be encountered by FBG sensors in a range of applications. These experiments showed that a simple linear temperature coefficient is insufficient to represent Bragg wavelength shifts over relatively modest temperature ranges. The effect is $\sim 20$-pm deviation from linearity, equivalent to a $2{ }^{\circ} \mathrm{C}$ error over the temperature range $-30{ }^{\circ} \mathrm{C}$ to $80^{\circ} \mathrm{C}$, and $\sim 35$-pm deviation for an extended temperature range of $-70{ }^{\circ} \mathrm{C}$ to $80{ }^{\circ} \mathrm{C}$, which hence is not negligible. The measured nonlinearity is consistent with Ghosh's model of the temperature dependence of the thermo-optic coefficient of silica glass, which predicts a quadratic term in the Bragg wavelength shift as a function of temperature and is in very good agreement with the observations. To our knowledge this is the first report of the use of this model to explain the nonlinear temperature response of a FBG. We have also characterized the strain response of three FBGs spanning 1524-1573 nm. The measured wavelength change was observed to be linear with applied strain, and the wavelength-normalized strain sensitivity was measured to be $0.81 \times 10^{-6} \mu \varepsilon^{-1}$.

Table 2. Linear Regression Analysis of Strain Response of a FBG Array for 1524-, 1549-, and 1573-nm FBGs

\begin{tabular}{ccc}
\hline FBG $\lambda_{\varepsilon=0}(\mathrm{~nm})$ & Sensitivity $\left(\mathrm{pm} \mu \varepsilon^{-1}\right)$ & $\begin{array}{c}(1 / \lambda)(\Delta \lambda / \Delta \varepsilon) \\
\left(\times 10^{-6} \mu \varepsilon^{-1}\right)\end{array}$ \\
\hline $1524.16354 \pm 0.00014$ & $1.22881 \pm 0.00013$ & 0.9999993 \\
$1548.86369 \pm 0.00023$ & $1.25087 \pm 0.00021$ & 0.9999980 \\
$1572.97662 \pm 0.00053$ & $1.26992 \pm 0.00050$ & 0.8076 \\
0.8073 & 0.999887 \\
\hline
\end{tabular}


The authors acknowledge provision of funding by the UK Engineering and Physical Sciences Research Council and the provision of a studentship by BAE Systems, through the Co-operative Awards in Science and Engineering scheme, for G. M. H. Flockhart, R. R. J. Maier acknowledges support from AWE plc.

\section{References}

1. Y. J. Rao, "Recent progress in applications of in-fibre Bragg grating sensors," Opt. Lasers Eng. 31, 297-324 (1999).

2. G. P. Brady, K. Kalli, D. J. Webb, D. A. Jackson, L. Reekie, and J. L. Archambault, "Simultaneous measurement of strain and temperature using the first- and second-order diffraction wavelengths of Bragg gratings," IEE Proc. Optoelectron. 144, 156-161 (1997).

3. D. R. Hjelme, L. Bjerkan, S. Neegard, J. S. Rambech, and J. V. Aarsnes, "Application of Bragg grating sensors in the characterization of scaled marine vehicle models," Appl. Opt. 36, 328-336 (1997).

4. K. Sugden, L. Zhang, J. A. R. Williams, R. W. Fallon, L. A. Everall, K. E. Chisholm, and I. Bennion, "Fabrication and characterization of bandpass filters based on concatenated chirped fiber gratings," J. Lightwave Technol. 15, 1424-1432 (1997).

5. G. Ghosh, "Temperature dispersion of refractive-indexes in some silicate fiber glasses," IEEE Photon. Technol. Lett. 6, 431-433 (1994).

6. S. Gupta, T. Mizunami, T. Yamao, and T. Shimomura, "Fiber Bragg grating cryogenic temperature sensors," Appl. Opt. 35, 5202-5205 (1996).

7. M. B. Reid and M. Ozcan, "Temperature dependence of fiber optic Bragg gratings at low temperatures," Opt. Eng. 37, 237 240 (1998).

8. S. W. James, R. P. Tatam, A. Twin, M. Morgan, and P. Noonan, "Strain response of fibre Bragg grating sensors at cryogenic temperatures," Meas. Sci. Technol. 13, 1535-1539 (2002).

9. A. Hidayat, Q. Wang, P. Niay, M. Douay, B. Poumellec, F. Kherbouche, and I. Riant, "Temperature-induced reversible changes in spectral characteristics of fiber Bragg gratings," Appl. Opt. 40, 2632-2642 (2001).

10. G. M. H. Flockhart, R. McBride, W. N. MacPherson, J. D. C. Jones, K. E. Chisholm, L. Zhang, I. Bennion, I. Read, and P. D. Foote, "Application of Hilbert transforms to high resolution strain and temperature characterisation of fibre Bragg grating sensors," in 14th International Conference on Optical Fiber Sensors, A. G. Mignani and H. C. Lefèvre, eds., Proc. SPIE 4185, 25-28 (2000).

11. O. V. Butov, K. M. Golant, and I. V. Nikolin, "Ultra-thermoresistant Bragg gratings written in nitrogen-doped silica fibres," Electron. Lett. 38, 523-525 (2002).

12. T. S. Priest, K. T. Jones, G. B. Scelsi, and G. A. Woolsey, "Thermal coefficients of refractive index and expansion in optical fibre sensing," in Optical Fiber Sensors, Vol. 16 of 1997 OSA Technical Digest Series (Optical Society of America, Washington, D.C., 1997), Paper OWC41.

13. T. Toyoda and M. Yabe, "The temperature dependence of the refractive indices of fused silica and crystal quartz," J. Phys. D Appl. Phys. 16, L97-L100 (1983).

14. F. M. Haran, J. K. Rew, and P. D. Foote, "A strain-isolated fibre Bragg grating sensor for temperature compensation of fibre Bragg grating strain sensors," Meas. Sci. Technol. 9, 1163-1166 (1998).

15. G. Ghosh, "Temperature dispersion of refractive-indexes in some silicate fiber glasses," IEEE Photon. Technol. Lett. 6, 431-433 (1994).

16. G. Ghosh, "Model for the thermo-optical coefficients of some standard optical glasses," J. Non-Cryst. Solids 189, 191-196 (1995).

17. D. A. Flavin, R. McBride, and J. D. C. Jones, "Short-scan interferometric interrogation and multiplexing of fibre Bragg grating sensors," Opt. Commun. 170, 347-353 (1999).

18. K. B. Rochford and S. D. Dyer, "Demultiplexing of interferometrically interrogated fiber Bragg grating sensors using Hilbert transform processing," J. Lightwave Technol. 17, 831-836 (1999).

19. R. Kashyap, Fiber Bragg Gratings (Academic Press, San Diego, Calif., 1999).

20. J. M. Jewell, "Thermooptic coefficients of some standard reference material glasses," J. Am. Ceram. Soc. 74, 1689-1691 (1991).

21. J. Matsuoka, N. Kitamura, S. Fujinaga, T. Kitaoka, and H. Yamahita, "Temperature-dependence of refractive-index of $\mathrm{SiO}_{2}$ glass,” J. Non-Cryst. Solids 135, 86-89 (1991).

22. G. Ghosh, M. Endo, and T. Iwasaki, "Temperature-dependent Sellmeier coefficients and chromatic dispersions for some optical-fiber glasses," J. Lightwave Technol. 12, 1338-1342 (1994). 